\title{
Strates
}

STRATES Matériaux pour la recherche en sciences sociales

$11 \mid 2004$

Jeune recherche, la vitalité d'un laboratoire

\section{Pasteurs et agro-pasteurs de la steppe algérienne}

Enquête sur la rgion de Djebel Amour

\section{Riad BENSOUIAH}

\section{CpenEdition}

Journals

Édition électronique

URL : http://journals.openedition.org/strates/478

DOI : $10.4000 /$ strates.478

ISSN : $1777-5442$

Éditeur

Laboratoire Ladyss

\section{Édition imprimée}

Date de publication : 1 janvier 2004

ISSN : 0768-8067

Référence électronique

Riad BENSOUIAH, "Pasteurs et agro-pasteurs de la steppe algérienne », Strates [En ligne], 11 | 2004, mis en ligne le 17 janvier 2005, consulté le 08 septembre 2020. URL : http://journals.openedition.org/ strates/478; DOI : https://doi.org/10.4000/strates.478

Ce document a été généré automatiquement le 8 septembre 2020

Tous droits réservés 


\title{
Pasteurs et agro-pasteurs de la steppe algérienne
}

\author{
Enquête sur la rgion de Djebel Amour
}

\section{Riad BENSOUIAH}

1 La steppe algérienne, bande longitudinale qui s'étale des frontières est aux frontières ouest sur une superficie d'environ 20 millions d'hectares, a toujours été un territoire d'élevage dédié en premier lieu aux ovins.

2 Traditionnellement, la transhumance était pratiquée par les pasteurs afin d'offrir à leurs troupeaux des pâturages d'été en se déplaçant au nord tellien ${ }^{1}$ pour échapper au climat aride de la steppe, et des pâturages d'hiver au sud de la steppe afin d'éviter aux animaux le froid rude des zones steppiques. Le pastoralisme était donc fondé sur deux grands déplacements annuels par lesquels les éleveurs s'efforçaient de préserver leurs cheptels et de permettre la régénération des pâturages.

3 Aujourd'hui, les pratiques du pastoralisme ont changé. Ces changements sociaux, économiques, organisationnels ou même naturels, ont eu des effets non seulement sur la vie des pasteurs, mais aussi et surtout sur le milieu naturel. Dans toutes les steppes du monde, on parle de dégradation des parcours ${ }^{2}$ et bien évidemment la steppe algérienne ne fait pas exception. 


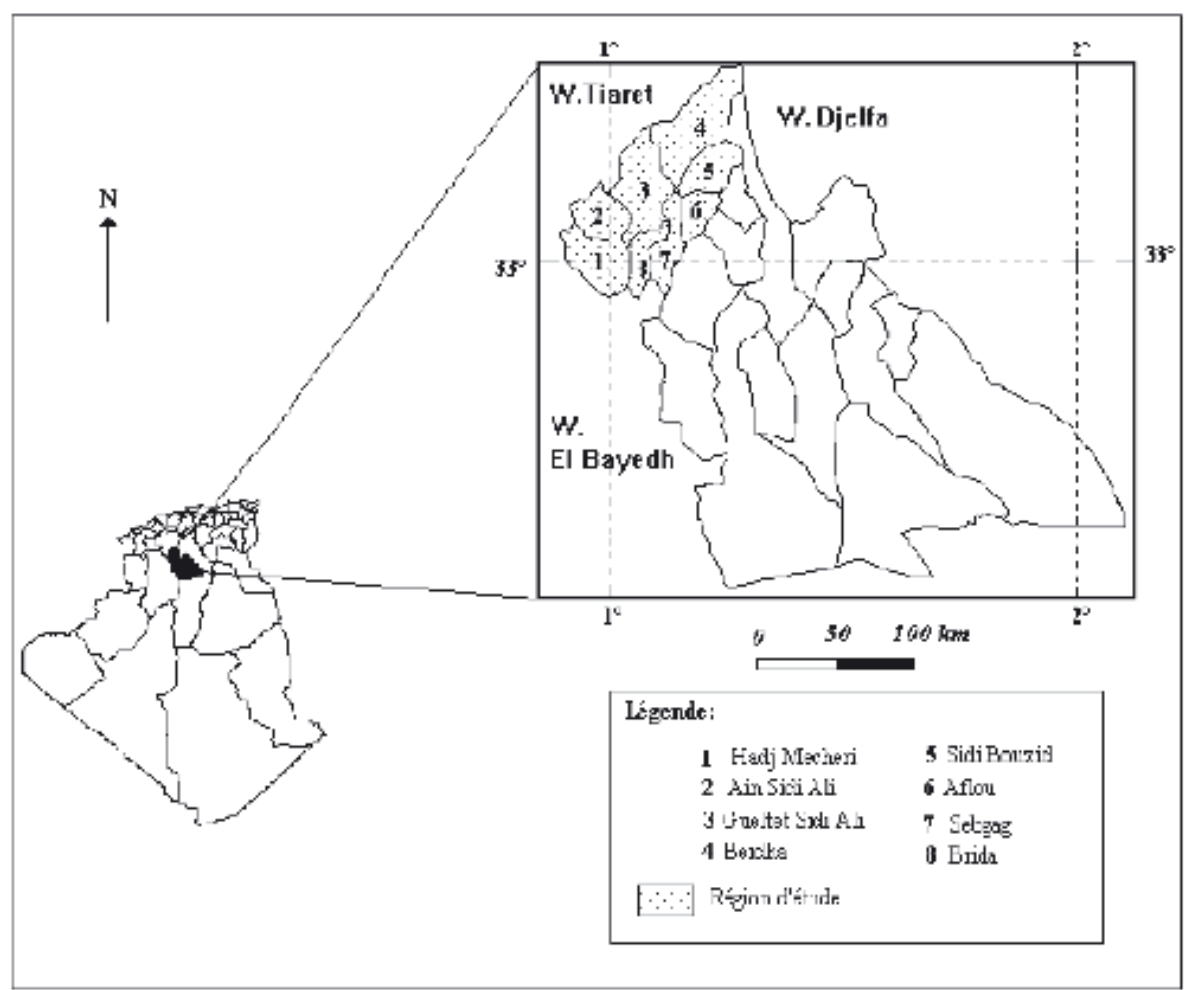

Source : Carte élaborée par R. Bensouiah.

4 La dégradation des qualités écologiques (réduction de la biodiversité) et paysagères (extension du paysage désertique), ainsi que la réduction de la superficie des parcours, principal facteur de production sur lequel se basait l'activité, ne peuvent plus être négligées.

5 Toutefois, les changements de nature différente interagissent de telle façon qu'il est difficile d'affirmer que la dégradation du milieu serait à l'origine de la paupérisation constatée dans la steppe algérienne, car l'inverse est tout aussi vrai. Seule une analyse dynamique, tentant de retracer les différentes phases d'évolution de la société et des espaces pastoraux algériens, pourrait permettre de sortir de cette difficulté. Cette étude de cas tente d'y contribuer, en s'efforçant d'identifier quelques-unes des stratégies développées par les pasteurs et les agro-pasteurs de la steppe algérienne pour faire face aux transformations de leur territoire et de leur mode de vie.

Données socio-économiques sur la région

1. Répartition générale des terres en 1994.

\begin{tabular}{|l|l|l|}
\hline & Superficie (Hectare) & Part (\%) \\
\hline \hline Surface agricole utile (SAU) & 25939 & 5,63 \\
\hline \hline Pacages et parcours & 365743 & 79,34 \\
\hline Autres & 51678 & 11,21 \\
\hline
\end{tabular}




\begin{tabular}{|l|l|l|}
\hline Totale terres agricoles & 443360 & 96,17 \\
\hline \hline Exploitations forestières & 17640 & 3,83 \\
\hline \hline Superficie totale de la région & 461000 & 100 \\
\hline
\end{tabular}

Source : DSA de la Wilaya de Laghouat, 1995.

2. Évolution de la population de la région entre 1977 et 1994.

\begin{tabular}{|l|l|l|l|l|l|l|l|}
\hline & 1966 & 1977 & 1987 & 1991 & 1994 \\
\hline \hline Population totale & 9296 & 52500 & 69834 & 82583 & 90308 \\
\hline \hline Indice d'évolution & 100 & 564,76 & 751,22 & 888,71 & 971,47 \\
\hline \hline Part dans la population de la wilaya (\%) & 21,43 & 35,37 & 32,88 & 32,83 & 32,44 \\
\hline
\end{tabular}

Source : DPAT de la wilaya de Laghouat

Présentation de l'enquête

6 Notre étude repose sur les résultats d'une enquête que nous avons réalisée dans la région du Djebel Amour, plus précisément centrée sur le massif du Djebel Amour (Atlas Saharien), localisé au nord de la wilaya de Laghouat, environ à $300 \mathrm{~km}$ à vol d'oiseau au sud d'Alger. Cette région s'étend sur une superficie de $4810 \mathrm{~km}^{2}$. Du point de vue administratif, elle comprend trois dairates et huit communes ${ }^{3}$.

7 Physiquement, elle est constituée par deux zones naturellement bien distinctes : la zone des Hauts Plateaux steppiques, située au nord et à caractère agro-pastoral-alfatier, et celle de l'Atlas saharien, située au sud et à caractère sylvo-agro-pastoral.

L'enquête a été menée durant le printemps 1996 auprès d'un échantillon représentatif d'exploitations pastorales, agricoles et agropastorales de six communes de la région.

Dans un premier temps, nous avons constitué des listes des exploitations de chaque commune par enquête auprès des Assemblées populaires communales et des notables de chaque Douar ${ }^{4}$. Au total, 3536 exploitations ont ainsi été identifiées sur les six communes, chaque exploitation étant caractérisée par un certain nombre de critères : commune d'appartenance, caractéristiques juridiques (nom patronymique, filiation du chef...), paramètres techniques (Superficie Agricole Utile, nombre de brebis reproductrices, présence d'un tracteur agricole...).

Dans un second temps, nous avons classé ces exploitations en six catégories :

Tableau 1. Classification des exploitations recensées.

\begin{tabular}{|l|l|l|l|l|}
\hline Catégorie & SAU & Brebis & Tracteur & $\begin{array}{l}\text { Nombre d'exploi- } \\
\text { tations recensées }\end{array}$
\end{tabular}




\begin{tabular}{|l||l|l|l|l|l|l|l|}
\hline 1 & + & + & + & 117 & Agro-éleveur mécanisé \\
\hline \hline 2 & + & + & - & 2491 & Agro-éleveurs non mécanisé \\
\hline \hline 3 & & & & & & & \\
\hline \hline
\end{tabular}

Pour la catégorie 3, notons que l'éleveur, par définition sujet à une mobilité dans l'espace, peut ne pas disposer de superficie agricole. En revanche l'agro-pasteur, du fait de sa pratique d'autres activités et de la nature de son système de production, dispose de SAU et éventuellement de tracteurs.

12 La catégorie 5 a été divisée en cinq sous-catégories, en fonction du nombre de brebis reproductrices:

Tableau 2. Répartition des éleveurs non mécanisés selon la taille du cheptel reproducteur

\begin{tabular}{|l|l|l|}
\hline Sous-catégorie & Nombre de brebis reproductrices & Nombre d'exploitations \\
\hline \hline 51 & 1 à 20 têtes & 107 \\
\hline \hline 52 & 21 à 50 têtes & 103 \\
\hline \hline 53 & 51 à 100 têtes & 44 \\
\hline 54 & 101 à 200 têtes & 40 \\
\hline \hline 55 & Supérieur ou égal à 201 têtes & 21 \\
\hline
\end{tabular}

13 Nous avons ensuite procédé à un tirage au hasard d'un échantillon de 200 exploitations, conforme à la répartition par catégories de la population mère (le taux de sondage atteint étant de 5,66 \%). Toutefois, lors de l'enquête, il s'est avéré qu'une dizaine d'entre elles avaient connu récemment des changements de caractéristiques ou s'avéraient peu représentatives. Afin d'atténuer le risque d'introduction de biais, nous les avons éliminées et avons finalement retenu 190 exploitations.

14 Par le biais de questionnaires, puis du traitement des données recueillies, nous nous sommes ensuite efforcés de cerner les principaux aspects des dynamiques en cours dans la région, et en particulier d'identifier les stratégies des pasteurs et agro-pasteurs. Quelques-uns de ces aspects sont présentés ici5.

Le mode de mise en valeur des ressources : un décalage entre disponibilités et besoins

Si l'on suit Ph. Daget et M. Gordon, « le pastoralisme en tant qu'activité est le moyen le plus efficace pour utiliser les ressources sur les terres sèches ou marginales. En temps 
normal, les pasteurs nomades sont souvent mieux nantis que les agriculteurs sédentaires. Ils peuvent déplacer leurs bêtes pour suivre les pluies ou les conduire aux pâturages saisonniers établis. Mais ils sont souvent les premières victimes du stress environnemental prolongé, par exemple la sécheresse $»^{6}$.

La principale ressource des zones steppiques reste «le parcours». Espace commun selon son statut juridique, il constitue le principal facteur de production. Les parcours occupent une grande part de la superficie des zones steppiques. Leur étendue ainsi que leurs caractéristiques naturelles les dédient beaucoup plus à l'activité pastorale qu'à d'autres activités économiques, d'où l'attribut « vocation pastorale ».

Dans la région de Djebel Amour, ces parcours représentent $29 \%$ de la superficie totale.

Le couvert végétal est dominé principalement par deux espèces, l'alfa (stipa tenacissima) et l'armoise blanche (artmisea herba alba) dont le nom local est chih, qui constituent l'essentiel de l'offre en pâturage pour les espèces animales domestiques.

18 Toutefois, si les déplacements que pratiquaient les pasteurs permettaient - en théorie du moins - la régénération des parcours et la reprise du couvert végétal, cette dynamique a été rompue récemment du fait de l'accroissement du cheptel dans la région, comme partout ailleurs dans la steppe algérienne, et de la sédentarisation simultanée des hommes induisant une surcharge de certains parcours.

L'accroissement du cheptel de la région a en effet été considérable puisqu'on a enregistré une croissance annuelle moyenne de 5716 têtes, correspondant à une progression des effectifs de 124590 têtes en 1955 à 351432 têtes en 1994 ( $C f$. graphe 1).

Le surpâturage est ainsi devenu chronique, engendrant une dégradation continue des parcours. La charge pastorale est le meilleur indicateur pour estimer ce degré de dégradation. Si la charge qui permettait l'équilibre a pu être estimée en 1982 à 0,25 têtes/ha (Boukhobza, 1982), soit 4 hectares pour un ovin, celle constatée à Djebel Amour est quatre fois plus élevée, atteignant 0,96 têtes/ha. Elle est même beaucoup plus élevée dans les zones les plus accessibles, notamment dans celle des hauts plateaux steppiques: par exemple, dans la commune d'Ain Sidi Ali, cette charge représente 11 fois la norme citée plus haut.

21 En 1955, on enregistrait 0,37 têtes/ha, ce qui conférait à la région une situation proche de la «normale ». La charge pastorale de la région est ensuite passée de 0,55 têtes/ha en 1987 à 0,96 têtes/ha en 1994.

Graphe 1. Évolution du cheptel ovin dans la région de Djebel Amour entre 1955 et 1994 Source : J. Depois (1957) et DSA de la wilaya de Laghouat.

Tableau 3. évolution de la charge pastorale par commune entre 1987 et 1994 (tête/ha)

\begin{tabular}{|l||c|c|c|}
\hline & 1987 & 1991 & 1994 \\
\hline \hline Aflou & 0,39 & 1,70 & 1,22 \\
\hline \hline Sidi Bouzid & 0,28 & 2,16 & 0,29 \\
\hline \hline Sebgag & 1,41 & 2,09 & 1,59 \\
\hline
\end{tabular}




\begin{tabular}{|l|l|l|l|}
\hline Gueltet Sidi Saâd & 0,29 & 0,78 & 0,52 \\
\hline \hline Ain Sidi Ali & 0,96 & 2,5 & 2,86 \\
\hline \hline Beïdha & 0,36 & 1,83 & 0,46 \\
\hline \hline Hadj Mecheri & 0,75 & 1,37 & 1,18 \\
\hline \hline Brida & 1,01 & 0,78 & 1,65 \\
\hline Région & 0,55 & 1,40 & 0,96 \\
\hline
\end{tabular}

Source : Calculé par l'auteur d'après les données de la DSA de Laghouat.

Notons que la régression constatée au niveau de certaines communes entre 1991 et 1994 est due au fait que leurs parcours sont dans un état avancé de dégradation et n'attirent plus les troupeaux qui se dirigent vers les parcours moins dégradés de Brida et plus encore d'Ain Sidi Ali : d'où la forte augmentation de la charge pastorale dans ces deux communes.

L'ensemble des communes affiche des charges pastorales élevées par rapport à la norme admise. Toutefois, à la lecture du tableau, deux catégories de communes peuvent être distinguées: celles qui accusent une progression quasi continue de leur charge, induisant une intensification du processus de dégradation des parcours, et celles qui affichent une croissance moyenne eu égard à l'ensemble.

La privatisation de fait de certains parcours

En rupture avec le mode de production ancestral, on est passé d'un élevage transhumant, fondé sur la mobilité des hommes et des animaux, à un élevage fixe basé sur la complémentation de l'alimentation des animaux. Le début de ce passage, d'un mode de conduite ancestral à un mode de conduite nouveau dont les pasteurs ignorent les règles mais surtout les conséquences sur leur environnement, remonte à peu près à un siècle. Toutefois, c'est à partir des années soixante-dix que l'on assiste à une amplification des modifications observées. La plus grande partie des éleveurs et agroéleveurs enquêtés situe dans ces années soixante-dix le début de la complémentation de l'alimentation des animaux, tout comme les défrichements des terres de parcours et la sédentarisation.

Le recours à l'agriculture peut être interprété de différentes manières. Pour les plus riches, c'est une façon de s'approprier de l'espace, pour les pauvres, une source de revenus secondaires en l'absence d'emplois salariés. Les comportements observés obéissent ainsi à des motifs économiques.

Pour asseoir leur stratégie de délimitation-appropriation de terres, les plus riches s'appuient sur un droit coutumier qui, s'agissant d'une terre labourée, prévoit l'interdiction de la traverser. Ce procédé leur procure la pleine jouissance des terres de parcours dont ils ont pris possession.

27 Le libre accès pour tout un chacun, jadis règle commune à l'ensemble des acteurs sociaux, a été remis en cause en peu de temps, au début des années soixante-dix, par le développement d'une pratique appelée $E l$ Gdel, qui consiste à relier au minimum trois 
lots de céréales de quelques traits de labours, de façon à délimiter un parcours à l'intérieur d'une terre et à empêcher les autres d'y pénétrer?

Ceux qui disposent de moyens matériels leur permettant de labourer leurs terres et jouissent d'une position sociale importante s'arrogent ainsi l'exclusivité de l'accès à certains parcours.

De l'avis de nos enquêtés, depuis que cette pratique est appliquée, il y aurait de moins en moins de parcours d'accès libre pour tous. Le quart d'entre eux mentionnent l'existence de lieux, dans leurs communes, auxquels ils pouvaient auparavant accéder, mais qui leur sont aujourd'hui interdits, essentiellement du fait d'El Gdel. $19 \%$ voient dans cette pratique un choix inévitable du simple fait qu'elle est devenue chose courante. De fait, un paysage de parcours labourés à la déchaumeuse est offert à tout curieux dès que celui-ci quitte les principaux axes routiers...

Figure 1. Mode de pratique d'El Gdel

Pourtant, en théorie, cette pratique est illicite car le labour des terres de parcours est interdit par la réglementation - d'où la difficulté à l'atteindre. Lors des enquêtes, seuls 36 enquêtés ont reconnu s'y livrer (tandis qu'ils étaient 86 , soit près de la moitié de l'échantillon, à affirmer que leurs voisins s'y adonnaient...). Les chiffres obtenus sousestiment certainement l'ampleur de cette pratique. Pour l'ensemble de notre échantillon, la superficie qui lui est consacrée s'élèverait en 1996 à 305 ha, répartis entre les diverses catégories de telle manière que les éleveurs les plus aisés possèdent en moyenne la superficie la plus importante : si l'on considère la superficie moyenne en Gdel par enquêté, elle est la plus élevée pour un agriculteur mécanisé, suivi d'agroéleveurs mécanisés.

Tableau 3. Répartition de la superficie laissée en Gdel par catégorie d'enquêtés (1996)

\begin{tabular}{|c|c|c|c|c|}
\hline Catégorie & $\begin{array}{l}\text { Nombre total } \\
\text { d'enquêtés } \\
\text { dans } \\
\text { l'échantillon }\end{array}$ & $\begin{array}{l}\text { Nombre d'enquêtés qui } \\
\text { déclarent pratiquer } \mathrm{El} \\
\text { Gdel }\end{array}$ & $\begin{array}{l}\text { Superficie } \\
\text { totale } \\
\text { (hectare) }\end{array}$ & $\begin{array}{l}\text { Superficie } \\
\text { moyenne (hectare/ } \\
\text { enquêté) }\end{array}$ \\
\hline $\begin{array}{l}\text { Agro-éleveurs } \\
\text { mécanisés }\end{array}$ & 8 & 4 & 56 & 14 \\
\hline $\begin{array}{l}\text { Agro-éleveurs } \\
\text { non mécanisés }\end{array}$ & 133 & 29 & 224 & 7,72 \\
\hline $\begin{array}{l}\text { Agriculteurs non } \\
\text { mécanisés }\end{array}$ & 33 & 1 & 3 & 3 \\
\hline $\begin{array}{l}\text { Éleveurs non } \\
\text { mécanisés }\end{array}$ & 15 & 1 & 2 & 2 \\
\hline $\begin{array}{l}\text { Agriculteurs } \\
\text { mécanisés }\end{array}$ & 1 & 1 & 20 & 20 \\
\hline
\end{tabular}




\begin{tabular}{|l|l|l|l|l|}
\hline Total & 190 & 36 & 305 & 8,47 \\
\hline
\end{tabular}

Source : Données de l'enquête.

31 Pour les agro-éleveurs non mécanisés, les terres concernées sont de mauvaise qualité et/ou se situent sur des parcours très éloignés: elles ne sont pas convoitées par les agro-éleveurs mécanisés qui préfèrent opter pour des parcelles de bonne qualité, proches de leurs lieux d'habitation.

Les pasteurs les plus démunis, en revanche, sont pratiquement exclus de cette pratique : leur position sociale ne leur permet guère de s'y livrer et ils sont dépourvus de tracteurs susceptibles d'opérer la délimitation concrète sur le terrain.

Une activité agropastorale au devenir aléatoire

Le niveau de vie de ceux qui s'y adonnent est un élément essentiel du devenir de l'activité agropastorale. Par manque de données statistiques sur le revenu des enquêtés, nous avons rapproché, pour cerner leur niveau de vie, plusieurs critères (facteurs de production possédés, pluri-activité ou mono-activité du ménage, habitat possédé, etc.) Par exemple, un éleveur ou agro-éleveur habitant sur les parcours et possédant une maison " secondaire » à Aflou ne peut être qu'« aisé » : en plus d'une maison, la plupart des grands éleveurs habitant le centre urbain de la région possèdent ainsi un commerce tenu par l'un de leurs fils.

34 À l'exception des agro-éleveurs mécanisés, qui jouissent d'un bon niveau de vie, la majorité de nos enquêtés ont un niveau de vie moyen. Les plus pauvres se trouvent dans les éleveurs non-mécanisés des sous-catégories 51 et 52 (disposant de moins de 50 brebis) et dans les agro-éleveurs non mécanisés qui ont le moins de cheptel.

Tableau 4. Répartition des catégories d'acteurs, selon leur niveau de vie

\begin{tabular}{|c|c|c|c|c|}
\hline \multicolumn{2}{|l|}{ CATEGORIES } & \multicolumn{3}{|c|}{ NIVEAU DE VIE } \\
\hline & & Bon & Moyen & Mauvais \\
\hline \multicolumn{2}{|l|}{ Agro-éleveurs mécanisés } & & & \\
\hline \multicolumn{2}{|c|}{ Agro-éleveurs non mécanisés } & & & \\
\hline \multicolumn{2}{|c|}{ Agriculteurs non mécanisés } & & & \\
\hline & C51 & & & \\
\hline Éleveurs non mécanisés & C52 & & & \\
\hline & C53 & & & \\
\hline & C54 & & & \\
\hline
\end{tabular}


Agriculteurs mécanisés

Source : Données de l'enquête.

Ceux qui sont considérés comme "aisés » représentent une faible proportion de la population enquêtée : ils sont au nombre de 8. La ressource « espace » et/ou moyens de production, sont détenus par une minorité d'éleveurs tandis que la majorité d'entre eux sont confrontés à une paupérisation accentuée par un combat sans merci pour la maîtrise des parcours. Cette paupérisation se manifeste essentiellement par une décapitalisation qui touche principalement les éleveurs non mécanisés, et par la sédentarisation $^{8}$ des nomades qui préfèrent s'installer dans les bidonvilles aux alentours d'Aflou que de rester sous la tente sur les parcours, d'autant que ce mode de vie n'est plus toléré, surtout par les femmes. Les pasteurs nomades se considèrent ainsi comme les oubliés de la modernisation.

La dimension du cheptel est évidemment étroitement liée au niveau de vie : la catégorie la moins nombreuse, disposant du meilleur niveau de vie, possède également le plus de cheptel:

Tableau 5. Importance du cheptel dans les différentes catégories d'enquêtés

\begin{tabular}{|l|l|l|l|l|}
\hline & $\begin{array}{l}\text { Catégorie plus } \\
\text { aisée }\end{array}$ & $\begin{array}{l}\text { Catégorie } \\
\text { moyenne }\end{array}$ & $\begin{array}{l}\text { Catégorie } \\
\text { démunie }\end{array}$ & plus \\
\hline \hline Nombre d'enquêtés & 8 & 128 & 54 & 190 \\
\hline $\begin{array}{l}\text { Cheptel ovin total } \\
\text { (tête) }\end{array}$ & 1642 & 6786 & 3438 & \\
\hline \hline Cheptel/enquêté (tête) & 205 & 53 & 63 & 48 \\
\hline
\end{tabular}

Source : Données de l'enquête

Si les enquêtés de la catégorie regroupant les plus démunis possèdent plus de cheptel que ceux de la catégorie moyenne, cela tient au fait que les premiers pratiquent essentiellement l'élevage tandis ceux de la catégorie moyenne pratiquent aussi l'agriculture. On ne peut pour autant voir dans l'élevage une source de pauvreté, puisque beaucoup d'enquêtés ont fait fortune grâce à l'élevage...

La concentration du cheptel est observable pour l'ensemble de la steppe algérienne. En 1982, M. Boukhobza notait déjà que $10,7 \%$ des éleveurs, possédant des troupeaux de plus de 100 têtes, détenaient environ $68,5 \%$ du cheptel ovin steppique.

Tableau 6. Concentration du cheptel ovin dans la steppe algérienne en 1982

\begin{tabular}{|l|l|l|}
\hline & Propriétaires (\%) & Cheptel (\%) \\
\hline \hline Moins de 10 ovins & 64,4 & 7,6 \\
\hline
\end{tabular}




\begin{tabular}{|c|c|c|}
\hline 10 à 50 ovins & 15,1 & 8,7 \\
\hline 50 à 100 ovins & 9,8 & 15,2 \\
\hline 100 à 300 ovins & 6,9 & 25,4 \\
\hline 300 ovins et plus & 3,8 & 43,1 \\
\hline Total & 100 & 100 \\
\hline
\end{tabular}

Source : M. Boukhobza. d'équilibre. Pour les éleveurs et les agro-éleveurs de Djebel Amour, la catégorie moyenne représente $67 \%$ de l'ensemble de la population étudiée. Elle se caractérise par la possession d'un facteur de production unique, le cheptel, car la terre est dans la plupart des cas en propriété collective, sauf pour les agriculteurs de la catégorie 4 qui possèdent des superficies agricoles en propriété privée négligeable, destinées au maraîchage. L'objectif des enquêtés de cette catégorie est d'avoir un niveau de vie décent tout en maintenant une activité agropastorale, en s'appuyant éventuellement sur des activités capables de générer un revenu d'appoint : travail saisonnier chez des tiers, travail salarié de l'un des membres du ménage en ville ou dans les agglomérations limitrophes.

Figure 2. Dynamique des catégories d'éleveurs et d'agro-éleveurs de Djebel Amour

Source : Élaboré par l'auteur.

Cette catégorie intermédiaire semble évoluer dans deux directions différentes. Certains capitalisent un maximum de cheptel, de moyens humains, matériels et financiers et rejoignent les plus aisés : ils sont peu nombreux à y parvenir. Les autres s'inscrivent dans un cercle vicieux de paupérisation et rejoindront progressivement la catégorie des plus démunis. La catégorie intermédiaire pourrait ainsi être appelée à disparaitre. Si 
c'était le cas, les différenciations sociales accrues pourraient avoir des conséquences dépassant largement le champ écologique et économique.

Conclusion saisonnier.

Chez les pasteurs et les agro-pasteurs, la dynamique est gérée par la décapitalisation pour les plus démunis et la thésaurisation du cheptel pour les plus aisés; en revanche chez les agriculteurs, elle est gérée par le gain et/ou la perte des superficies agricoles ${ }^{9}$.

Il y a deux types de dynamiques: une endogène et l'autre exogène. La dynamique endogène se matérialise par des transformations au sein d'une même catégorie ou le passage d'une catégorie à une autre tout en restant au sein de l'activité mère - l'activité agropastorale. La dynamique exogène, quant à elle, se caractérise par une sortie de la sphère de l'activité agropastorale. Elle peut avoir deux sens différents : soit l'abandon de l'activité et le recours au salariat pour les plus démunis, soit, pour les plus aisés, la thésaurisation et la capitalisation pour investir dans d'autres domaines, notamment le commerce. Le graphique suivant met en évidence l'importance de la source mixte :

Graphe 2 : Source de revenu des enquêtés

Source : Données de l'enquête.

Ainsi, $56 \%$ des enquêtés assurent leurs revenus en recourant à des activités extraagricoles diverses ou combinent le revenu de l'activité agropastorale et une retraite, une pension ou d'autres aides familiales. Ceux dont les ressources sont uniquement fondées sur un revenu assuré par l'élevage ou l'agriculture sont les moins nombreux. La tendance est à la diversification des activités et au recours aux revenus extra-agricoles.

Le temps de la gestion collective des ressources naturelles est révolu. L'ère actuelle porte plutôt un individualisme dénié.

4 Les changements constatés dans l'activité pastorale ont eu des effets variables en fonction de la catégorie à laquelle appartiennent les pasteurs ou agro-pasteurs. Les plus « aisés » ont su les mettre à profit pour se développer. En revanche, les plus démunis subissent les effets de ces changements qui ne font que les inscrire dans un cycle de paupérisation et de marginalisation.

La course vers la maîtrise des ressources et les vifs conflits sur les terres de parcours obéissent à deux stratégies différentes. Les plus aisés tentent d'avoir plus de moyens et de pouvoir social afin de maximiser leurs profits, les plus démunis tentent de subsister grâce au maintien d'un élevage extensif et d'une agriculture en sec, s'appuyant sur quelques hectares de terres collectives et sur le recours au salariat et au travail

4 L'intégration au marché n'est que le résultat de la combinaison de facteurs favorables qui permettent aux plus aisés une ouverture sur les autres régions du pays par les biais de la commercialisation et de l'approvisionnement en facteurs de production. La marginalisation des plus démunis résulte quant à elle de la conjonction de facteurs limitant le développement de l'activité agropastorale et/ou pastorale, par le biais d'une décapitalisation progressive et d'une paupérisation - dans une dynamique marquée par une diminution des catégories moyennes qui ne parviennent pas à se reproduire.

50 La situation est telle que l'accumulation des problèmes sociaux, économiques et environnementaux pourrait compromettre la survie des populations et de l'écosystème dans la steppe algérienne. 


\section{BIBLIOGRAPHIE}

Abaab, A., Bedrani, S., Bourbouze, A. \& Chiche, J., 1995. « Les politiques agricoles et la dynamique des systèmes agro-pastoraux au Maghreb ». Options Méditerranéennes, Série B no 14 (Agriculture maghrébine à l'aube de l'an 2000), Ciheam/Iam Montpellier.

Bedrani, S., 1994. Algérie, les développement des zones de parcours. Étude Banque mondiale, Washington.

Bedrani, S. \& Bensouiah, R., 2000. « La relation homme - environnement dans la steppe algérienne : cas de la région de Djebel Amour ». Communication au séminaire international MEDENPOP 2000. Jerba, Tunisie du 25 au 28 octobre 2000.

Bensouiah, R., 1997. La dégradation de parcours steppiques : étude de cas sur la région de Djebel Amour. Thèse de Magister. INA, Alger.

Boukhobza, M., 1982. L'agro-pastoralisme traditionnel en Algérie : de l'ordre tribal au désordre colonial. OPU, Alger.

Capot Rey, R., 1942. « Le nomadisme pastorale dans le Sahara français ». Travaux de l'IRS, Vol. 1, Alger.

Daget, Ph. \& Gordon, M., 1995. Pastoralisme : troupeaux, espaces et sociétés. Hattier -AUPELF. UREF, Paris.

Despois, J., 1957. Le Djebel Amour. Éd. PUF, Paris.

Emery, R., 1998. « Hight-Rentability Pastoralism Versus Risk-Averse Pastoralism ». Journal of Environment \& Development, Vol. 7, no 4.

Manger Leif, O., 1999. From the Mountains to the Plains: the Integration of the Lafofa Nuba into Sudanese Society. OSSREA, Addis Abeba.

Manger Leif, O. \& Abdelghafar, A. M., 2000. Pastoralists and Environment: Experience from the Greater Horne of Africa. Procuding of the Regional Workshop on African Drylands. OSSREA, Addis Abeba.

\section{NOTES}

1.Le Tell est la zone du Nord de l'Algérie, celle qu'on appelle aussi l'Algérie utile car c'est la seule région où l'agriculture intensive est possible.

2.Cf. les travaux de Ph. Daget et al., 1995 ; Emery R., 1998 et Manger L. et al., 2000.

3.En Algérie, l'organisation administrative distingue wilaya, daïra et commune (équivalent en France de département, arrondissement et commune)

4.Une tribu est constituée de plusieurs Douar.

5.L'ensemble des résultats a été présenté dans une thèse soutenue en novembre 2003 à l'université de Paris X, sous la direction d'Hugues Lamarche : Dynamique socioéconomique et culturelle des espaces pastoraux algériens. Cas de la région de Djebel Amour. NDR.

6.Daget Ph. et Gordon M. (1995) : Pastoralisme: troupeaux, espaces et sociétés. Hattier AUPELF.UREF, Paris.

7.Cette pratique peut prendre d'autres formes et d'autres expressions, selon le contexte. Au Maroc par exemple, sous un autre nom, elle se pratique exactement selon 
le même principe, mais au lieu de lots de céréales, on relie des tentes de membres de la famille de l'éleveur.

8.À la question relative aux raisons de la sédentarisation, nos enquêtés ont été unanimes pour dire que l'objectif était la recherche de conditions de vie meilleures. 9.Or, nous savons maintenant que l'accroissement de la superficie agricole possédée oblige le recours à El Gdel.

\section{RÉSUMÉS}

Sur la base des résultats d'une enquête menée au cours du printemps 1996, l'auteur s'efforce de cerner quelques-unes des dynamiques à l'œuvre au sein des différentes catégories de pasteurs et d'agro-pasteurs de la région de Djebel Amour : face aux évolutions récentes, les plus "aisés » adoptent des stratégies visant à une maximisation des profits, tandis que les moins nantis s'efforcent avant tout d'assurer le maintien de leur niveau de vie.

Basing himself on the results of an enquiry led in the spring of 1996, the author tries to determine some of the dynamics at work within the different categories of pastors and agropastors from the region of Djebel Amour. Confronted to recent evolutions, the wealthiest adopt strategies aiming at a maximization of their benefits, while the less rich struggle to preserve their living standards.

\section{INDEX}

Index géographique : Algérie

Mots-clés : Agriculture

\section{AUTEUR}

RIAD BENSOUIAH

Sociologue. Doctorat, Ladyss, université de Paris 10, bensouia@hotmail.com 